\title{
MMSE-Based Amplifying Relay Matrix for Noncooperative AF Wireless Relay Network under Power Constraints
}

\author{
Kanghee Lee \\ Department of Electrical \\ Engineering and Computer Science \\ Wichita State University \\ Wichita, Kansas 67220
}

\author{
Hyuck M. Kwon \\ Department of Electrical \\ Engineering and Computer Science \\ Wichita State University \\ Wichita, Kansas 67220
}

\author{
Zuojun Wang \\ Department of Electrical \\ Engineering and Computer Science \\ Wichita State University \\ Wichita, Kansas 67220
}

\begin{abstract}
This paper derives analytically an optimum amplifying relay matrix using the minimum mean square error (MMSE) criteria for a noncooperative amplify-and-forward (AF) distributed relay network. Global and local power constraints are included in the analysis. And a one-source-one-destination node pair and $N$-relay network is considered. Because the relays are noncooperative, this paper exploits the diagonal property in an amplifying relay matrix for the optimum matrix derivation. Then, this paper claims that the bit error rate of the AF network using the proposed amplifying relay matrix is significantly better than that of the AF network with the other existing amplifying matrices.
\end{abstract}

Index Terms-Amplify-and-forward, minimum mean square error, amplifying relay matrix, power constraint.

\section{INTRODUCTION}

In recent years, the importance of relay node roles in wireless networks has been increasing in the area of wireless communication. In relation to this, various relay schemes, such as nonregenerative amplify-and-forward (AF), regenerative decode-and-forward (DF), selective decode-and-forward (SDF), and compress-and-forward (CF), have been studied in [1]-[6]. The nonregenerative AF relay scheme employed in this current paper is a more popular and realistic protocol due to no required signal processing at the relay nodes for decoding and compressing procedures compared to the other three relay schemes. In other words, all relay nodes in the nonregenerative AF relay scheme only amplify their received signals from a source node and transmit the amplified signals to a destination node.

In addition, efficient amplifying relay matrices using different objective functions and power constraints have been sou-

This work was partly sponsored by the Army Research Office under DEPSCoR ARO Grant W911NF-08-1-0256, and by NASA under EPSCoR CAN Grant NNX08AV84A.

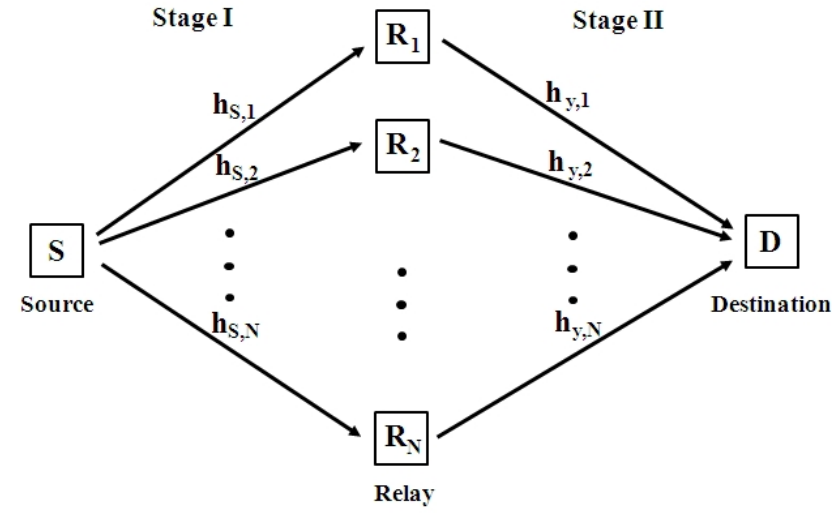

Fig. 1. Wireless relay network for $N$ relay nodes and a one-source-onedestination pair node under power constraints at the destination node.

ght for a single-input-single-output AF network [6]-[8]. In [6], the authors presented the AF relay strategy under the received signal power constraint at the destination node for a onesource-one-destination pair and $N$-relay node network. The advantage of using the power constraint at the destination node instead of the source node or the relay nodes is to accomplish a better performance by maximizing the received signal component power. A solution of closed form in (82) of [6] can only be achieved with an assumption of low-noise relay nodes. And, under this assumption, multiple approximations were used in [6]. Behbahani and Eltawil in [7] minimized the mean square error cost under the power constraint at an equalizer input. Recently, the authors in [8] derived an optimal relay factor for the one-source-one-destination pair and $N$-relay node network to minimize MSE under the power constraint at the destination node. However, Behbahani et al. in [7] and Behbahani and Eltawil in [8] employed the proportionality to solve the optimization problem as shown in (17) of [7] and in (15) of [8], respectively. However, in [9], [10], noncooperative distributed minimum mean square error (MMSE)-based AF 
relay schemes in wireless networks under the received power constraint at the destination node for single-input single-output with $N$ relay nodes was studied by the authors of this paper. Particularly, jamming environment and channel uncertainty were considered in [9]. While, broadband jamming and channel uncertainty were considered in [10]. However, in [9], [10], effects of noncooperative MMSE relay scheme under both local and global power constrains were not considered.

Therefore, as was done in [9], [10], to achieve performance better under a more realistic environment, compared to the previous schemes in [6]-[8], this current paper proposes a different minimum mean square error (MMSE) cost function under both local and global power constraints at the destination node without any assumptions and approximations made in [6]-[8] using the noncooperative strategy definition. In other words, this current paper focuses on only BER performance under no-jamming environment and no-node geometry. In particular, a diagonal optimum amplifying relay matrix will be derived using the objective function because the noncooperative distributed AF wireless relay network is considered. This current paper will compare BER performance for the two cases between the local power constraint and global power constraint using a diagonal optimum amplifying relay matrix.

The remaining paper is organized into five sections. Section II describes the overall system model and data transmission strategy. Section III and Section IV present the noncooperative diagonal optimum amplifying relay matrices based on the MMSE relay schemes, respectively, under a global power constraint and a local power constraint at the destination node. Section V shows the simulation results. Finally, Section VI concludes the paper.

Notation: Matrices and vectors are denoted, respectively, by uppercase and lowercase boldface characters (e.g., $\mathbf{A}$ and $\mathbf{a}$ ). The transpose, complex conjugate, inverse, and Hermitian of $\mathbf{A}$ are denoted, respectively, by $\mathbf{A}^{T}, \mathbf{A}^{*}, \mathbf{A}^{-1}$, and $\mathbf{A}^{H}$. An $n \times n$ identity matrix is denoted by $\mathbf{I}_{N}$. The expectation operator is $E[\cdot]$. Notations $|a|,\|\mathbf{a}\|$, and $\|\mathbf{A}\|_{F}$ denote the absolute value of $a$ for any scalar, 2-norm of $\mathbf{a}$, and Frobenius-norm of $\mathbf{A}$, respectively.

\section{SySTEM MODEL}

Figure 1 illustrates a wireless relay network consisting of a source node, a destination node, and $N$ noncooperative distributed relay nodes. There are two stages for data transmission where a source node broadcasts a signal $s$ to the relay nodes in Stage I and the relay nodes retransmit their signals received from a source node to a destination node in Stage II, as shown in Fig. 1. This paper considers the case where the direct link from a source node to a destination node is considerably weak and can be negligible. The perfect channel coefficient complex column vector $\mathbf{h}_{s} \in \mathbf{C}^{N \times 1}$ from the source node to the relay nodes and the perfect channel coefficient complex row vector $\mathbf{h}_{d} \in \mathbf{C}^{1 \times N}$ from the relay nodes to the destination node are, respectively, written as

$$
\mathbf{h}_{s}=\left[h_{s, 1}, h_{s, 2}, \cdots, h_{s, N}\right]^{T}
$$

and

$$
\mathbf{h}_{d}=\left[h_{d, 1}, h_{d, 2}, \cdots, h_{d, N}\right]
$$

where the channel coefficient $h_{s, i}, i=1, \cdots, N$, from the source node to the $i$-th relay node is the $i$-th element of $\mathbf{h}_{s}$, and the channel coefficient $h_{d, i}$, from the $i$-th relay node to the destination node is the $i$-th element of $\mathbf{h}_{d}$. Each channel coefficient $h_{s, i}$ and $h_{d, i}$ is assumed to be independent identically distributed zero mean circular complex Gaussian with unit variance and quasi-static Rayleigh fading in order for them to be constant during data transmission. The received signal complex column vector $\mathbf{r} \in \mathbf{C}^{N \times 1}$ at the relay nodes is written as

$$
\mathbf{r}=\mathbf{h}_{s} s+\mathbf{v}_{s}
$$

where $\mathbf{v}_{s} \in \mathbf{C}^{N \times 1}$ is a zero-mean complex additive white Gaussian noise vector with covariance matrix $\sigma_{v_{s}}^{2} \mathbf{I}_{N}$. For the relay noncooperation, it is assumed that all relay nodes cannot communicate their received signal information from the source node to each other. The processed signal complex column vector $\mathbf{x} \in \mathbf{C}^{N \times 1}$ at the relay node outputs is given by

$$
\mathbf{x}=\mathbf{F r}
$$

where $\mathbf{F} \in \mathbf{C}^{N \times N}$ is a diagonal amplifying relay matrix used by the relay nodes to increase performance at the destination node. The received complex signal $d \in \mathbf{C}^{1 \times 1}$ at the destination node can be written as

$$
d=\mathbf{h}_{d} \mathbf{x}+v_{d}
$$

where $v_{d} \in \mathbf{C}^{1 \times 1}$ is a zero-mean complex additive white Gaussian noise with variance $\sigma_{v_{d}}^{2}$. Substituting (4) into (5) and using (3), the received complex signal $d \in \mathbf{C}^{1 \times 1}$ at the destination node can be written as

$$
d=\mathbf{h}_{d} \mathbf{F} \mathbf{h}_{s} s+\mathbf{h}_{d} \mathbf{F} \mathbf{v}_{s}+v_{d}=\mathbf{f h}_{s} s+\mathbf{f} \mathbf{v}_{s}+v_{d}
$$

where $\mathbf{f}\left(\in \mathbf{C}^{1 \times N}\right) \triangleq \mathbf{h}_{d} \mathbf{F}=\left[f_{d_{1}}, \ldots, f_{d_{N}}\right]$ is defined as a linear transformation row vector to apply the MMSE between the received signal at the destination node and the transmitted signal at the source node in the next section. 


\section{Noncooperative MMSE Relay Scheme UndeR GLOBAL POWER CONSTRAINT}

This section focuses on the noncooperative distributed MMSE relay scheme in wireless networks under a global power constraint $\mathrm{P}$ at the destination node, where $\mathrm{P}$ is the total power usage available to all relay nodes in a network. In other words, this section presents the steps in how to design a diagonal amplifying relay matrix $\mathbf{F}_{1}$ under the global power constraint using a linear transformation row vector $\mathbf{f}=\mathbf{h}_{d} \mathbf{F}_{1}$ in (6). The minimizing mean square error criterion between the received signal $d$ at the destination node and the transmitted signal $s$ at the source node is used under a global power constraint at the destination node. The optimization can be written as

$$
\begin{gathered}
\mathbf{F}_{1}^{\dagger}=\arg \min _{F_{1}} J\left(\mathbf{F}_{1}\right) \\
\text { s.t. } \quad E\left[\left|\mathbf{h}_{d} \mathbf{x}\right|^{2}\right]=\mathrm{P}
\end{gathered}
$$

where the objective function $J\left(\mathbf{F}_{1}\right)$ using the second equation in (6) is written as

$$
\begin{aligned}
J\left(\mathbf{F}_{1}\right) & =E\left[|d-s|^{2}\right] \\
& =\sigma_{s}^{2} \mathbf{f h}_{s} \mathbf{h}_{s}^{H} \mathbf{f}^{H}+\sigma_{v_{s}}^{2} \mathbf{f f}^{H}-\sigma_{s}^{2} \mathbf{f h}_{s}-\sigma_{s}^{2} \mathbf{h}_{s}^{H} \mathbf{f}^{H}+\sigma_{s}^{2}+\sigma_{v_{d}}^{2} .
\end{aligned}
$$

Using (5) and the second equation in (6), the amount of global signal power at the destination node is written as

$$
\mathrm{P}=\sigma_{s}^{2}\left|\mathbf{f h}_{s}\right|^{2}+\sigma_{v_{s}}^{2}|| \mathbf{f}||_{2}^{2} .
$$

To solve a constrained optimization problem, a Lagrangian multiplier $\lambda$ in [11] is applied as

$$
L\left(\mathbf{F}_{1}, \lambda\right)=J(F)_{1}+\lambda\left(E\left[\left|\mathbf{h}_{d} \mathbf{x}\right|^{2}\right]-\mathrm{P}\right) .
$$

Differentiating $L\left(\mathbf{F}_{1}, \lambda\right)$ in terms of $\mathbf{f}$ using the properties of the derivative matrix [12] to (10), and employing the noncooperative scheme design, the $i$-th element of $\mathbf{f} \in \mathbf{C}^{1 \times N}$ is represented as

$$
f_{d_{i}}=h_{d, i} f_{i}=\frac{\sigma_{s}^{2} h_{s, i}^{*}}{(1+\lambda)\left(\sigma_{v_{s}}^{2}+\sigma_{s}^{2}\left\|\mathbf{h}_{s}\right\|_{2}^{2}\right)} .
$$

Using (11), the global power constraint at the destination node is written as

$$
\mathrm{P}=\sum_{i=1}^{N} \frac{\left(\sigma_{s}^{2}\left|h_{s, i}\right|^{2}+\sigma_{v_{s}}^{2}\right) \sigma_{s}^{4}\left|h_{s, i}\right|^{2}}{(1+\lambda)^{2}\left(\sigma_{v_{s}}^{2}+\sigma_{s}^{2}\left\|\mathbf{h}_{s}\right\|_{2}^{2}\right)^{2}} .
$$

Using (12), the Lagrangian multiplier $\lambda$ can be written as

$$
\lambda= \pm \sqrt{\frac{1}{\mathrm{P}} \sum_{i=1}^{N} \frac{\left(\sigma_{s}^{2}\left|h_{s, i}\right|^{2}+\sigma_{v_{s}}^{2}\right) \sigma_{s}^{4}\left|h_{s, i}\right|^{2}}{\left(\sigma_{v_{s}}^{2}+\sigma_{s}^{2}\left\|\mathbf{h}_{s}\right\|_{2}^{2}\right)^{2}}}-1 .
$$

Substituting (13) into (11), a diagonal optimum amplifying relay matrix $\mathbf{F}_{1}^{\dagger}$ for the power of entire relay nodes can be written as

$$
\mathbf{F}_{1}^{\dagger}=\operatorname{diag}\left(f_{1}, \ldots, f_{N}\right)
$$

where

$$
f_{i}=\frac{h_{s, i}^{*} \sqrt{\mathrm{P}}}{h_{d, i} \sqrt{\sum_{k=1}^{N}\left|h_{s, k}\right|^{2}\left(\sigma_{s}^{2}\left|h_{s, k}\right|^{2}+\sigma_{v_{s}}^{2}\right)}}, i=1, \ldots, N .
$$

This implies that the $i$-th relay needs to know only its own channel coefficient from the source to the $i$-th relay and from the $i$-th relay to the destination because the square root in the denominator of (15) is common and behaves as a normalization coefficient. Hence, $f_{i}$ plays a role as a matched filter for the source-to-relay link and a precoder for the $i$-th relay-to-the destination. Regardless of the sign of $f_{i}$, the same BER performance is achieved. Hence, the positive sign of $f_{i}$ is used in Section V.

For performance comparisons, the existing $\mathrm{AF}$ relay schemes proposed in [6]-[8] under their objective functions and power constraints for the one-source-one-destination and $N$-relay node noncooperative distributed wireless relay network are, respectively, presented in the rest of this section, and applied for simulation in Section V. In other words,

$$
\text { - } f_{i} \approx \frac{h_{s, i}^{*} h_{d, i}^{*} \sqrt{\mathrm{P}}}{\sqrt{\sum_{k=1}^{N} \frac{\left|h_{s, k}\right|^{2} \mid h_{\left.h_{,}\right|^{2}}}{\sigma_{s}^{2}\left|h_{s, k}\right|^{2}+\sigma_{v_{s}}^{2}}}\left(\sigma_{s}^{2}\left|h_{s, i}\right|^{2}+\sigma_{v_{s}}^{2}\right)} \text { in [6] }
$$

$$
\begin{aligned}
& \text { - } f_{i}=\frac{h_{s, i}^{*} h_{d, i}^{*} \sqrt{\mathrm{P}}}{\sigma_{r_{i}}^{2} \sqrt{\frac{\sigma_{v_{d}}^{2}}{\mathrm{P}}+\frac{\left|h_{d, i}\right|^{2} \sigma_{v_{s}}^{2}}{\sigma_{r_{i}}^{2}}} \sqrt{\sum_{k=1}^{N} \frac{\left|h_{s, k}\right|^{2}\left|h_{d, k}\right|^{2}}{\frac{\sigma_{v_{d}}^{2} \sigma_{r_{k}}^{2}}{\mathrm{P}}+\left|h_{d, k}\right|^{2} \sigma_{v_{s}}^{2}}}} \text { in [7] } \\
& \text { • } f_{i}=\frac{h_{s, i}^{*} h_{d, i}^{*} \sqrt{\mathrm{P}}}{\left|h_{d, i}\right|^{2} \sigma_{r_{i}}^{2} \sqrt{\frac{\sigma_{v_{d}}^{2}}{\mathrm{P}}+\frac{\sigma_{v_{s}}^{2}}{\sigma_{r_{i}}^{2}}} \sqrt{\sum_{k=1}^{N} \frac{\left|h_{s, k}\right|^{2}}{\frac{\sigma_{v_{d}}^{2} \sigma_{r}^{2}}{\mathrm{P}}+\sigma_{v_{s}}^{2}}}} \text { in [8]. }
\end{aligned}
$$

where $\sigma_{r_{i}}^{2}$ is the received signal power of the $i$-th relay node.

\section{NONCOOPERATIVE MMSE RELAY SCHEME UNDER LOCAL POWER CONSTRAINT}

In this section, a diagonal amplifying relay matrix $\mathbf{F}_{2}$ under local power constraint using a linear transformation row vector f is studied. The same minimizing MSE criterion between the received signal $d$ at the destination node and the transmitted signal $s$ at the source node under a local power constraint at the destination node can be written as,

$$
\mathbf{F}_{2}^{\dagger}=\arg \min _{F_{2}} J\left(\mathbf{F}_{2}\right)
$$




$$
\text { s.t. } E\left[\left|h_{d, i} x_{i}\right|^{2}\right]=p_{i}, i=1, \ldots, N \text {. }
$$

Similarly, using the second equation in (6), the objective function $J\left(\mathbf{F}_{2}\right)$ for the noncooperative scheme design can be written as

$$
\begin{aligned}
J\left(\mathbf{F}_{2}\right)= & \sigma_{s}^{2} \mathbf{f h}_{s} \mathbf{h}_{s}^{H} \mathbf{f}^{H}+\sigma_{v_{s}}^{2} \mathbf{f f}^{H}-\sigma_{s}^{2} \mathbf{f h}_{s}-\sigma_{s}^{2} \mathbf{h}_{s}^{H} \mathbf{f}^{H}+\sigma_{s}^{2}+\sigma_{v_{d}}^{2} \\
= & \sigma_{s}^{2}\left(\sum_{i=1}^{N} f_{d_{i}} h_{s, i}\right)\left(\sum_{i=1}^{N} f_{d_{i}}^{*} h_{s, i}^{*}\right)+\sigma_{v_{s}}^{2}\left(\sum_{i=1}^{N} f_{d_{i}} f_{d, i}^{*}\right) \\
& -\sigma_{s}^{2}\left(\sum_{i=1}^{N} h_{s, i}^{*} f_{d_{i}}^{*}\right)-\sigma_{s}^{2}\left(\sum_{i=1}^{N} f_{d_{i}} h_{s, i}\right)+\sigma_{s}^{2}+\sigma_{v_{d}}^{2}
\end{aligned}
$$

where $f_{d_{i}}$ is the $i$-th element of a linear transformation row vector $\mathbf{f} \in \mathbf{C}^{1 \times N}$ in (6). And the local power constraint of the $i$-th relay node can be written as

$$
p_{i}=\left|f_{d_{i}}\right|^{2}\left(\sigma_{s}^{2}\left|h_{s, i}\right|^{2}+\sigma_{v_{s}}^{2}\right)
$$

Similarly, the Lagrangian optimization can be written as

$$
L\left(\mathbf{F}_{2}, \lambda_{i}\right)=J\left(\mathbf{F}_{2}\right)+\sum_{i=1}^{N} \lambda_{i}\left(E\left[\left|h_{d, i} x_{i}\right|^{2}\right]-p_{i}\right)
$$

Differentiating $L\left(\mathbf{F}_{2}, \lambda_{i}\right)$ with respect to $f_{d_{i}}$ and $\lambda_{i}$, respectively, and using the noncooperative scheme design, the $i$-th element of the linear transformation row vector $\mathbf{f}$ can be written as

$$
f_{d_{i}}=\frac{\sigma_{s}^{2} h_{s, i}^{*}(1-\beta)}{\lambda_{i}\left(\sigma_{s}^{2}\left|h_{s, i}\right|^{2}+\sigma_{v_{s}}^{2}\right)+\sigma_{v_{s}}^{2}}
$$

where $\beta \in \mathbf{R}^{1 \times 1}=\sum_{k=1}^{N} f_{d_{k}}^{*} h_{s, k}^{*}$. Substituting (23) into (21), the Lagrangian multiplier $\lambda_{i}$ is obtained as

$$
\begin{aligned}
\lambda_{i}= & \frac{1}{\sigma_{s}^{2}\left|h_{s, i}\right|^{2}+\sigma_{v_{s}}^{2}} \times \\
& {\left.\left[ \pm \sqrt{p_{i}^{-1}\left(\sigma_{s}^{2}\left|h_{s, i}\right|^{2}(1-\beta)^{2}\left(\sigma_{s}^{2}\left|h_{s, i}\right|^{2}+\sigma_{v_{s}}^{2}\right)\right.}\right)-\sigma_{v_{s}}^{2}\right] }
\end{aligned}
$$

Therefore, a diagonal optimum amplifying relay matrix $\mathbf{F}_{2}^{\dagger}$ under each relay node power constraint can be written as

$$
\mathbf{F}_{2}^{\dagger}=\operatorname{diag}\left(f_{1}, \ldots, f_{N}\right)
$$

where

$$
f_{i}=\frac{h_{s, i}^{*} \sqrt{p_{i}}}{h_{d, i} \sqrt{\left|h_{s, i}\right|^{2}\left(\sigma_{s}^{2}\left|h_{s, i}\right|^{2}+\sigma_{v_{s}}^{2}\right)}}, \quad i=1, \ldots, N
$$

Similarly, the positive sign of $f_{i}$ is used in Section $\mathrm{V}$ because the identical BER performance is generated regardless of the sign of $f_{i}$. Using (26), the definition of $\beta$ becomes

$$
\beta=\sum_{i=1}^{N} f_{d_{i}}^{*} h_{s, i}^{*}=\sum_{i=1}^{N} \frac{ \pm\left|h_{s, i}\right| \sqrt{p_{i}}}{\sqrt{\sigma_{s}^{2}\left|h_{s, i}\right|^{2}+\sigma_{v_{s}}^{2}}} .
$$

\section{Simulation Results}

In order to evaluate the BER performance of the nonregenerative AF relay scheme in a noncooperative distributed wireless network under global and local power constraints using the proposed diagonal optimum amplifying relay matrices, the Monte-Carlo simulation is performed. All simulation results are for a one-source-one-destination pair network with a different number of noncooperative distributed relay nodes, $N=2,4,6$. It is assumed that all relay nodes with only one antenna are located at equidistance between the source and destination node. The general case with an arbitrary relay node geometry can be analyzed by using a simple cosine law. The ideally received signal at the destination node is assumed to be a 4-ary quadrature amplitude modulation (4QAM) symbol under global and local power constraints with unity power. The channel column vector $\mathbf{h}_{s}$ and the channel row vector $\mathbf{h}_{y}$ are generated from independent complex Gaussian random variables with zero mean and unity variance. It is also assumed that all relay nodes have the same noise power as the noise power at the destination, i.e., $\sigma_{v_{s}}^{2}=\sigma_{v_{y}}^{2}$. For fair BER comparisons with other diagonal amplifying relay matrices in [6]-[8], the same simulation parameters and conditions are used.

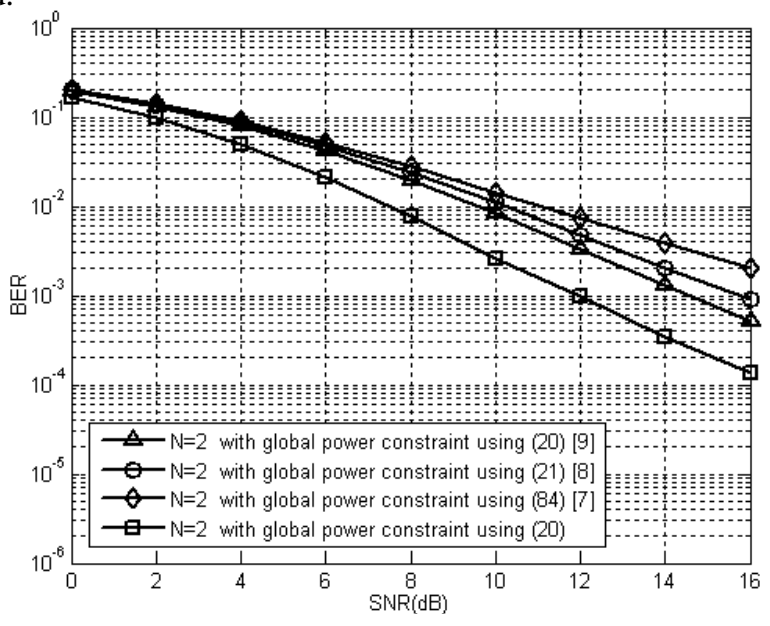

Fig. 2. BER performance comparisons of the proposed $N=2$ noncooperative distributed relay networks and the existing ones in [6], [7], and [8] under a global power constraint.

Fig. 2 shows BER performance comparisons of $N=2$ noncooperative distributed relay nodes using the proposed amplifying relay coefficient in (20) under the global power constraint, and the existing different diagonal amplifying relay matrices in (20) of [8], (21) of [7], and (84) of [6]. It is observed that, in spite of the noise inclusion at the destination node when the diagonal amplifying relay matrix is derived, the proposed one shows outstanding BER performance under the global power constraint. For example, using the proposed diagonal optimum amplifying relay matrix in (20) can show 
at least 3.5 $\mathrm{dB}$ improvement in SNR over others in [6]-[8] at $\mathrm{BER}=10^{-3}$. The SNR is defined to be $\sigma_{s}^{2} / \sigma_{v_{s}}^{2}$.

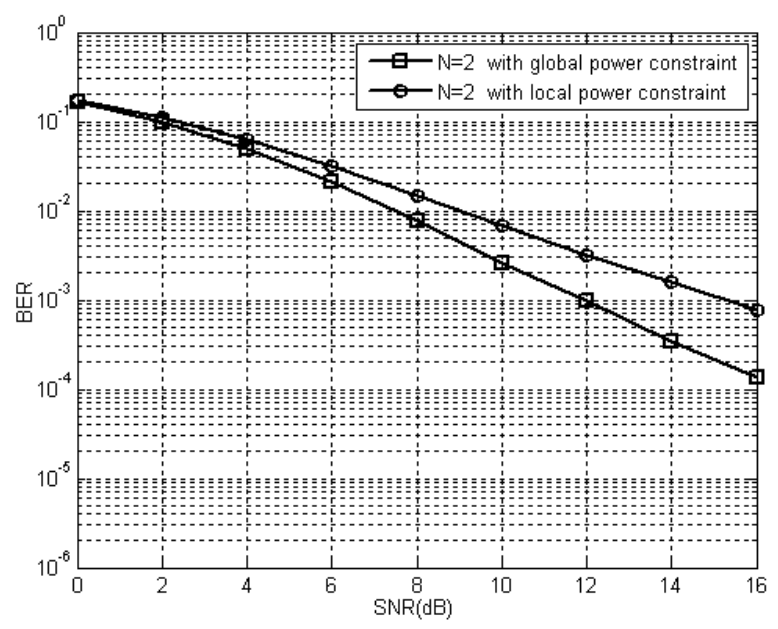

Fig. 3. BER performance of the proposed $N=2$ noncooperative distributed relay networks under either a global or a local power constraint.

Fig. 3 also shows BER performance of $N=2$ noncooperative distributed relay nodes under either the proposed global or local power constraints. It is found that the diagonal amplifying relay matrix of the global power constraint achieves a significantly better BER performance than that of the local power constraint. At $\mathrm{BER}=10^{-3}$, the global power constraint case can be $3 \mathrm{~dB}$ better than the local one.

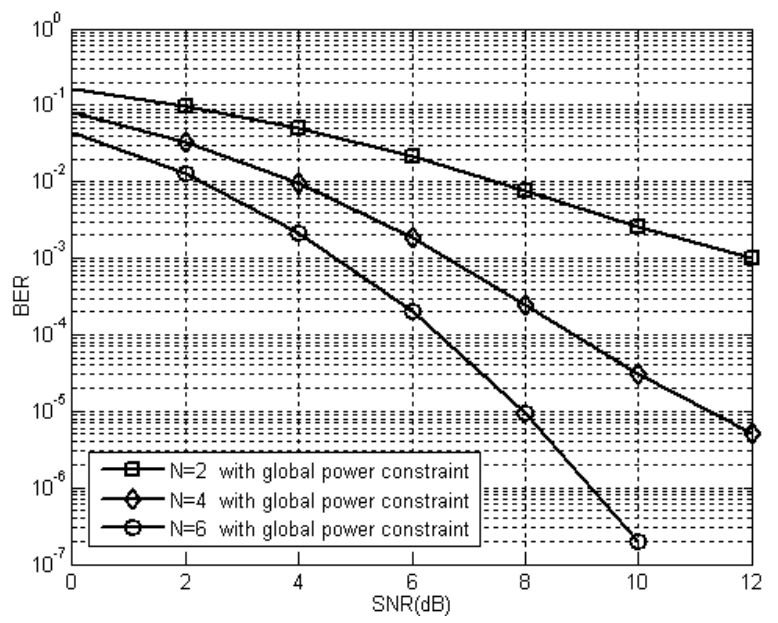

Fig. 4. BER performance of the proposed noncooperative distributed relay network under a global power constraint using $N=2,4$, and 6 number of relay nodes.

Fig. 4 shows BER performance of the proposed scheme under the global power constraint with different $N=2,4$, and 6 . It is observed that the BER performance gets improved significantly as the number of relay nodes increases. For example, the $N=6$ case can show approximately $2 \mathrm{~dB}$ and $7 \mathrm{~dB}$ improvement over the $N=4$ and $N=2$ case at $\mathrm{BER}=10^{-3}$, respectively.

\section{CONCLUSiON}

The diagonal optimum amplifying relay matrices using the MMSE were derived and proposed for the noncooperative distributed wireless relay networks under either a global or a local power constraint. A nonregenerative AF strategy for an $N$ relay node and one-source-one-destination pair network was considered. And the MSE between the received signal at the destination node and the transmitted signal at the source node was minimized under the power constraints at the destination node.

It was observed that BER performance of the proposed diagonal amplifying relay matrix improves significantly than the other existing ones in [6]-[8]. In addition, it was found that the diagonal amplifying relay matrix under a global power constraint accomplishes a significantly better BER performance than the one under a local power constraint. Finally, BER performance was found to be enhanced as the number of relay node increases.

\section{REFERENCES}

[1] T. Wang, G. B. Giannakis, and R. Wang, "Smart regenerative relays for link-adaptive cooperative communications," IEEE Transactions on Communications, vol. 56, no. 11, pp. 1950-1960, Nov. 2008.

[2] A. Saadani and O. Traoré, "Orthogonal or non-orthogonal amplify and forward protocol: How to cooperate?" IEEE WCNC 2008, Las Vegas, NV, Mar. 2008, pp. 368-373.

[3] J. Laneman, D. N. C. Tse, and G. W. Wornell, "Cooperative diversity in wireless networks: Efficient protocols and outage behaviour," IEEE Transactions on Information Theory, vol. 50, no. 12, pp. 3062-3080, Dec. 2004.

[4] S. Lee and S. Chung, "When is compress-and-forward optimal?" IEEE ITA 2010, San Diego, CA, Jan. 2010.

[5] G. Kramer, M. Gastpar, and P. Gupta, "Cooperative strategies and capacity theorems for relay networks," IEEE Transactions on Information Theory, vol. 51, no. 9, pp. 3037-3063, Sep. 2005.

[6] N. Khajehnouri and A. H. Sayed, "Distributed MMSE relay strategies for wireless sensor networks," IEEE Transactions on Signal Processing, vol. 55, no. 7, pp. 3336-3348, Jul. 2007.

[7] A. S. Behbahani, R. Merched, and A. M. Eltawil, "Optimizations of MIMO relay networks," IEEE Transactions on Signal Processing, vol. 56, no. 10, pp. 5062-5073, Oct. 2008.

[8] A. S. Behbahani and A. M. Eltawil, "Amplify-and-forward relay networks under received power constraint," IEEE Transactions on Wireless Communications, vol. 8, no. 11, pp. 5422-5426, Nov. 2009.

[9] K. Lee, H. M. Kwon, Y. Ding, Y. Ibdah, Z. Wang, and Y. Bi, "Node geometry and broadband jamming in noncooperative relay networks under received power constraint," IEEE 34th SARNOFF 2011, Princeton, NJ, May 3-4, 2011.

[10] Y. Ibdah, H. M. Kwon, K. Lee, Z. Wang, Y. Bi, and M. Jo, "Broadband jamming and channel uncertainty for noncooperative wireless relay networks under received power constraint," IEEE AMS 2011, Manila, Philippines, May 23-27, 2011.

[11] S. Boyd and L. Vandenberghe, Convex Optimization. Cambridge, U.K: Cambridge University Press, 1985.

[12] R. A. Horn and C. R. Johnson, Matrix Analysis. 1st ed., Cambridge, MA: Cambridge University Press, 1985. 